\title{
OBSERVATIONS ON TRACHOMATOUS ENTROPION AND ECTROPION*
}

\author{
BY \\ EDWARD ZAGORA \\ Menelik II Hospital, Addis Ababa, Ethiopia $\dagger$
}

THE sequelae of cicatricial entropion with trichiasis due to trachoma are still numbered among the most important causes of serious visual impairment in Ethiopia. Less frequent is trachomatous ectropion. Fortunately, each of these conditions can be permanently corrected when the proper surgical techniques are applied with adequate accuracy.

\section{Trachomatous Entropion with Trichiasis}

The procedure presented by Stallard (1958) as "tarsal paring and eversion" has, in my experience, been most satisfactory and I believe that it is the best technique to be adopted in any area of the world where the incidence of trachomatous entropion is still high. In the course of 559 operations of "tarsal paring and eversion" which I have performed in Addis Ababa from September, 1962, until the end of December, 1964, certain facts of interest have emerged. It is important, of course, to avoid under-correction. I have observed two causes of what could be termed "pseudounder-correction". One is corneal ectasia usually due to loss of corneal substance following ulceration, where the cornea was giving way before the intra-ocular pressure and was bulging. In spite of the operative eversion of the lid margin the cilia continuously injured the bulging cornea. The second cause of failure was senile atrophy of the skin of the upper lid with over-development of the lid folds and hanging down of the lid. This, of course, has been corrected by cosmetic surgery.

One patient in this series (a female aged 53) was found to have a bilateral symmetrical lagophthalmos in each eye caused by complete entropion and trichiasis of both lower lids. The patient was unable to close the lids by voluntary effort and the lower part of each cornea was opaque (Fig. 1). The operation of "tarsal paring and eversion" performed on each lower lid corrected both the entropion and the lagophthalmos.
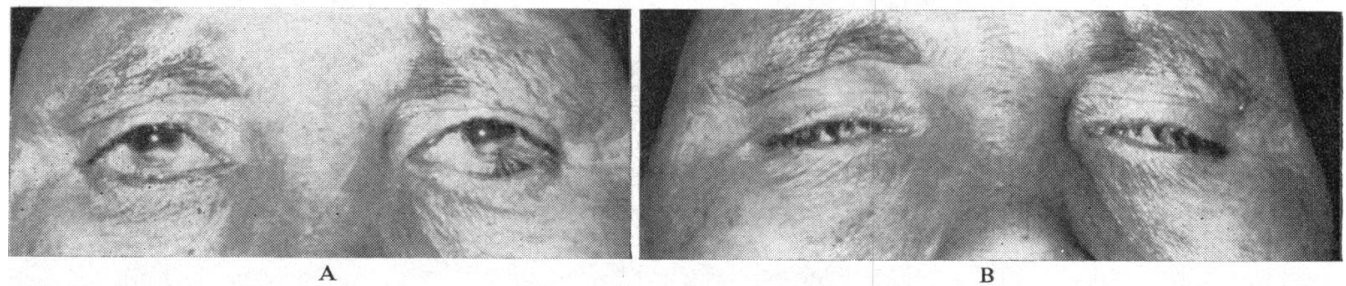

FIG. 1.-Trachomatous entropion of both lower lids with in-turning of the lashes (A), causing bilateral lagophthalmos (B).

* Received for publication April 29, 1965

† Present address: Internationella, Lund, Sweden. 
A survey follows of 559 eyes in 305 patients operated on for trachomatous entropion and trichiasis from September 1, 1962, to December 31, 1964 . The difference in frequency of operation on lids of the right and left eye is negligible. The percentage of the total number of lower lids requiring operation for entropion was strikingly small-45 (8 per cent.) lower lids compared with 514 (92 per cent.) upper lids (Table I).

TABLE I

COMPARISON OF INVOLVEMENT OF Eyes AND LiDS (559 LidS)

\begin{tabular}{l|c|c}
\hline \multirow{2}{*}{ Location of Involvement } & \multicolumn{2}{|c}{ Eyelids } \\
\cline { 2 - 3 } & No. & Per cent. \\
\hline Either lid of right eye & 283 & $50 \cdot 6$ \\
\hline Either lid of left eye & 275 & $49 \cdot 4$ \\
\hline Upper lids of either eye & 515 & $92 \cdot 3$ \\
\hline Lower lids of either eye & 45 & $8 \cdot 0$ \\
\hline
\end{tabular}

Noteworthy in this respect is the fact that the largest group in this series, 202 patients $(66.2$ per cent. of the total number), had entropion of both upper lids. It can be said that the operation for correction of trachomatous entropion of both upper lids is still the most frequently performed ophthalmic operation in this part of the world (Table II).

TABLE II

Topographical Distribution of Trachomatous Entropion in 305 Operated Cases

\begin{tabular}{l|r|c}
\hline \multicolumn{1}{c|}{ Topographical Distribution } & \multicolumn{2}{|c}{ Patients } \\
\cline { 2 - 3 } & No. & Per cent. \\
\hline Both lids of both eyes & 10 & $3 \cdot 2$ \\
Both right lids and left upper lid & 3 & $1 \cdot 0$ \\
Both left lids and right upper lid & 2 & $0 \cdot 7$ \\
Both lids of the right eye & 6 & $2 \cdot 0$ \\
Both lids of the left eye & 5 & $1 \cdot 7$ \\
Both upper lids & 202 & $66 \cdot 2$ \\
Both lower lids & 1 & $0 \cdot 3$ \\
Right upper lid & 37 & $12 \cdot 1$ \\
Right lower lid & 3 & $1 \cdot 0$ \\
Left upper lid & 32 & $10 \cdot 4$ \\
Left lower lid & 4 & $1 \cdot 4$ \\
\hline
\end{tabular}

The sex and age distribution (Fig. 2, opposite) revealed that there was a predilection for the female sex among the patients operated on-59.7 per cent. of females compared with 40.3 per cent. of males of the total number (305) of patients operated on. Moreover, the difference in relative frequency of males and females operated on was most striking in the fourth decade, in which females were operated on twice as often as males.

\section{Trachomatous Ectropion}

The shortening and bracing combination of Kuhnt's and Szymanowski's procedures is, in my experience, the method of choice for trachomatous ectropion, although as Fox (1958) rightly considers, it remains the classic operation for the repair of 


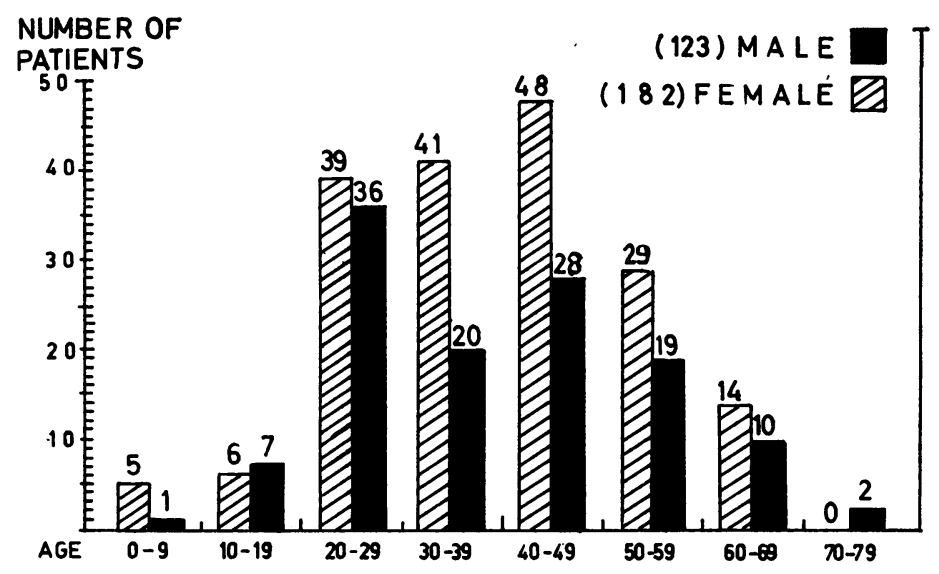

FIG. 2.-Trachomatous entropion with trichiasis-age and sex distribution.

atonic (senile) ectropion. This operation has given satisfactory results in cases of unilateral (Fig. 3) and bilateral (Fig. 4) trachomatous ectropion with total lid involvement.

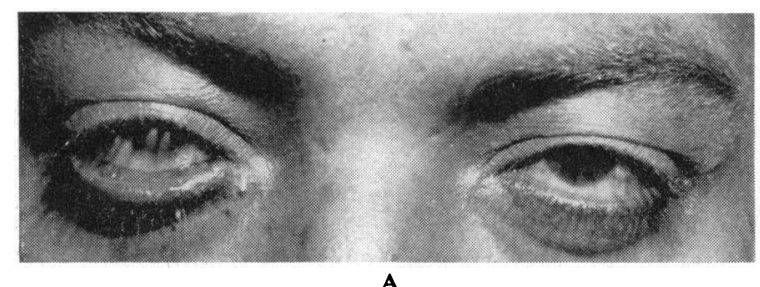

A

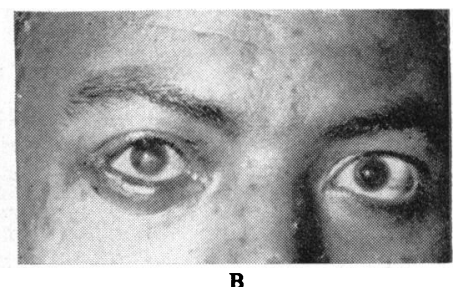

FIG. 3.-Trachomatous ectropion of right lower lid in a male aged 22 years (A), corrected by the Kuhnt-Szymanowski technique (B).
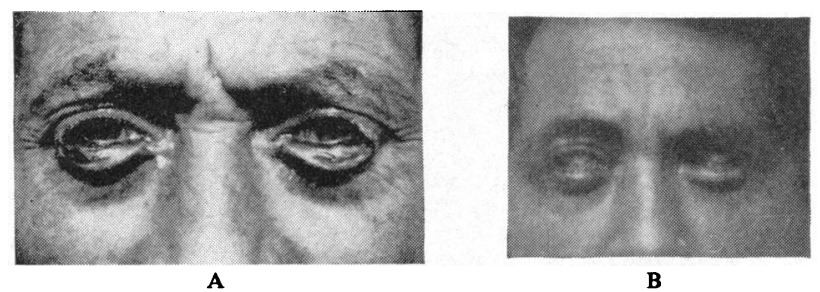

FIG. 4.-Trachomatous ectropion of both lower lids in a male aged 38 years $(A)$, and result of operation $(B)$.

It is interesting to note that an unusual case has been observed of a male aged 42 who had the following changes, all due to trachoma: an ankyloblepharon at the outer angle of each eye (with subsequent narrowing of the palpebral opening); an entropion with trichiasis of the right upper lid; an ectropion of the left lower lid and a slight eversion of the border of the left upper lid. The ankyloblepharon, which evidently developed during the healing process of trachoma involving the ciliary margins of the lids, was considerably more marked at the outer angle of the right eye 
than of the left eye (Fig. 5A, B). "Tarsal paring and eversion" procedure for the entropion of the right upper lid and the Kuhnt-Szymanowski technique for the ectropion of the left lower lid were performed at one sitting with a satisfactory result (Fig. 5C).

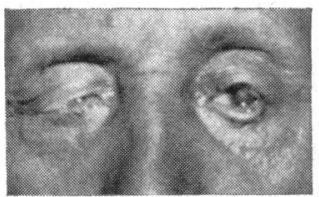

$\mathbf{A}$

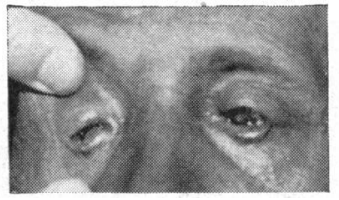

B

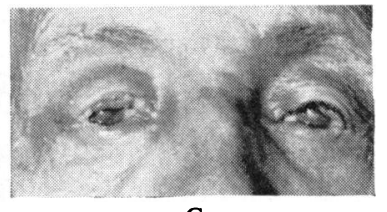

C

FIG. 5.-A, a case of bilateral partial ankyloblepharon with entropion and trichiasis of the right upper lid and ectropion of the left lower lid. B, the lids of the right eye forced open by the examiner in order to show the trichiasis. C, appearance after "tarsal paring and eversion" operation for entropion of the right upper lid and Kuhnt-Szymanowski procedure for ectropion of the left lower lid.

\section{Summary}

A pseudo-under-correction of trachomatous entropion may result in cases of corneal ectasia and also in cases of senile atrophy of the skin of the upper lid with over-development of the lid folds.

Entropion and trichiasis of both lower lids may, rarely, cause bilateral lagophthalmos, which disappears following surgical correction of the entropion.

The correction of trachomatous entropion of both upper lids is still the most frequently required ophthalmic operation in Ethiopia. The number of cases of entropion of the lower lids is relatively low.

A predilection for the female sex was found among the 305 patients operated on for trachomatous entropion, in which the highest numerical prevalence of females was in the fourth decade of life.

The technique of choice for correction of trachomatous entropion and trichiasis was found to be the operation of "tarsal paring and eversion", and for trachomatous ectropion the combination of Kuhnt's and Szymanowski's plastic procedures.

A case has been observed which shows that trachomatous changes may cause entropion with trichiasis in one eye and ectropion in the other eye of the same person.

\section{REFERENCES}

Fox, S. A. (1958). “Ophthalmic Plastic Surgery”, 2nd ed., p. 167. Grune and Stratton, New York. Stallard, H. B. (1958). "Eye Surgery”, 3rd ed., p. 191. Wright, Bristol. 